\title{
Professional Resources
}

\section{Software Reduces Run Times and Expands Nonlinear Material Modeling}

MSC Software Corp. has released March 2013.1, which provides faster nonlinear finite-element analysis (FEA) with new modeling options and increased ease of use. Performance improvements that take advantage of the modern multi-core processors are implemented. This may be used in conjunction with domain decomposition (DDM) and/or the parallel solution of equations of motion. When combined with DDM, this method provides two levels of parallelism: one at the domain level and one at the assembly level, enabling both designers and analysts to achieve improved productivity.

Tests show 5 to $8 \times$ faster element loop computation time and $4 \times$ faster total wall time for models with over 500,000 degrees of freedom on multi-core processors. Three new models represent the behavior of anisotropic incompressible materials such as rubber belts and biological materials, providing higher accuracy in simulations of reinforced elastomers. Also, users can now account for initial gaps or overlaps in a contact analysis to obtain accurate stress results.

For more information: MSC Software Corporation, 4675 MacArthur Court, Newport Beach, CA 92660; tel: 714/540-
8900; e-mail: americas.contact@mscsoftware.com; web: www. mscsoftware.com.

\section{Special Journal Issue Addresses Airworthiness and Fatigue}

A special issue of Science China Physics, Mechanics \& Astronomy contains twelve papers selected from the 2013 Proceedings of International Conference on Airworthiness and Fatigue, with authors from Australia, Russia, Greece, Italy, the United States, and China. The original conference manuscripts have been extended to journal style papers by the authors and have been subjected to peer review processing.

Some of the article topics include: adhesive bonding of composite aircraft structures, supersonic particle deposition to enhance structural integrity, very-high-cycle fatigue of in-service components, fatigue crack growth in fiber-metal laminates, and potential wells for classical acoustic waves.

For more information: SCIENCE CHINA Physics, Mechanics and Astronomy 2014, Volume 57, Issue 1: http://link.springer.com/journal/11433/57/1/page/1. 